Eds Jean-François Férard, Christian Blaise, Springer

ISBN 9789400750401

http://dx.doi.org/10.1007/978-94-007-5704-2 17

(c) Springer Science+Business Media Dordrecht

\title{
Bioavailability of contaminants
}

\author{
Catherine Gourlay-Francé ${ }^{1}$, Marie-Hélène Tusseau-Vuillemin ${ }^{2}$
}

1 Cemagref UR Hydrosystèmes et Bioprocédés. 1 rue Pierre-Gilles de Gennes, CS 10030, Antony, France

2 IFREMER, 155 rue Jean-Jacques Rousseau, Issy les Moulineaux, France

*: Corresponding authors : Catherine Gourlay-Francé, email address : catherine.gourlay@cemagref.fr ; Marie-Hélène Tusseau, email address : $\underline{\text { marie-helene.tusseau@ifremer.fr }}$

\section{Definition}

The ability of a chemical contaminant to interact with biological membranes of an organism and eventually have an adverse effect.

In the environment, contaminants are present in various chemical forms, depending both on their molecular properties and on the physical and chemical characteristics of the medium. The total concentration of a contaminant is not a reliable predictor of the actual exposure of organisms and its potential impacts on an ecosystem. Indeed, not all chemical forms are able to interact with biological membranes. The correct evaluation of the ecological risk due to chemicals lies in our ability to predict not only the total concentration in a medium, but particularly the bio-available concentration, which strongly depends on the contaminant molecular structure, the environment, but also on the exposed organisms.

\section{Historical overview, facts and background}

Ever since the work of Anderson et al. (1978), a number of studies have demonstrated that total dissolved metal concentrations are poorly correlated with observed effects on biological organisms. For most metals (e.g. $\mathrm{Cd}, \mathrm{Cu}, \mathrm{Ni}, \mathrm{Zn}, \mathrm{Pb}$ ), their complexation in solution leads to a reduction of biological effects. These experimental data have been explained via a common paradigm, called the Free lon Activity Model or FIAM (Morel 1983), according to which the biological effect induced by a metal is related to its free ion activity. This model has been validated by numerous laboratory experiments with several metals and organisms, as reviewed in Campbell (1995) and Batley et al. (2004). In this model, surface ligands located in the cell membrane allow cations to be internalized by the cell (Figure 1). The main hypotheses in the FIAM state that (i) there is an chemical equilibrium between metal in the environment and the metal bound to the surface ligand on the membrane, and hence the extent of the binding is governed by the free ion activity in the environment, (ii) internalization is the limiting step and (iii) the rate of internalization is proportional to the observed effect. In the 1990's, the same paradigm was applied to describe the effect of water chemistry on metal toxicity, leading to the Biotic Ligand Model (Paquin et al. 2002) also described 
in this book. The FIAM usually applies to divalent transition elements with the exception of $\mathrm{Hg}$ that may form lipophilic complexes (e.g. $\mathrm{HgCl}_{2}{ }^{0}$ ). The literature also points out that most experimental data that gave rise to the FIAM often poorly mimicked environmental conditions. In less contaminated media or in real and more complex environments, several studies suggest that some weakly complexed and rapidly exchangeable metals can contribute to the metal uptake into aquatic organisms under certain conditions (Slaveykova et al. 2003; Meylan et al. 2004; Ferreira et al. 2008). Nevertheless, the knowledge of the free ion concentration remains a cornerstone to assess the bioavailability of most metals $(\mathrm{Cu}, \mathrm{Cd}, \mathrm{Ni}, \mathrm{Zn}, \mathrm{Pb})$.

The bioavailability of hydrophobic organic contaminants is also strongly influenced by the chemical environment. Indeed, a large fraction of organic contaminants bind to organic matter, which affects their bioavailability. In the water column, most studies report the so-called protective effect of organic matter as the presence of dissolved organic matter in water reduces the bio-concentration of organic contaminants in numerous aquatic organisms (Haitzer et al. 1998). The generally-accepted assumption is that only the truly dissolved fraction of the chemical (also referred to as the "free fraction") is bioavailable to organisms that concentrate pollutants from water through direct contact and respiration (Landrum et al. 1985).

In soil and sediment, the bioavailability of contaminants is also strongly related to their partitioning between water and particles. As in water, the bioavailable organic compounds are generally assumed to be freely dissolved in porewater. This assumption has led to the "Equilibrium partitioning approach" (EqP) (Di Toro et al. 1991) in which the bioavailable concentration of a given contaminant is in equilibrium with both organic matter in the sediment and lipids of an exposed organism. The EqP approach allows predicting benthic contamination in various environments. Recently, the importance of contaminant desorption processes in sediments and soils has been pointed out. Indeed, some bound compounds may be released in water close to the biological membrane, and become available for accumulation. Semple et al. (2004) suggest that bioavailability and bioaccessibility should be distinguished, the bioavailable compound being freely available to cross the biological membrane at a given time (i.e. the freely dissolved compounds in porewater), whereas the bioaccessible compound is available to cross the membranes from the entire environment. Factors influencing the bioavailability of organic contaminants in soils and sediments have been extensively reviewed in Ehlers and Loibner (2006).

The bioavailability of metals in soil and sediment still remains poorly understood. Similarly to water, the FIAM and the BLM are commonly proposed (Di Toro et al. 2005), although there are few experimental validations because of the analytical difficulty of determining metal speciation in porewater. Indeed, it is difficult to collect large volumes of porewater and they are delicate to handle since they are rarely oxidized. As in the water column, there is growing evidence that the bioavailable fraction may also include rapidly exchangeable metals that desorb in the gut of the organism or in water due to the equilibrium displacement close to the biological membrane and hence contribute to metal uptake (Degryse et al. 2005). 


\section{Evaluation of the bioavailability of a contaminant}

The quantitative estimation of the bioavailable concentration of a contaminant is difficult, since it depends on the contaminant molecular structure, the environment and the exposed organisms (Figure 2). It can be estimated by a chemical measurement in the environment or through a biological response if the tested concentration induces measurable uptake or biological effects.

The physical-chemical measurement of bioavailability consists of measuring the different forms of the contaminant in the environment (for example, the distribution among aqueous, dissolved and particulate forms for organic compounds, or the evaluation of metal speciation) along with a hypothesis concerning the bioavailability of all chemical forms. This allows the differentiation of various contaminant pathways and related bioavailabilities either via ingestion or via direct contact (e.g., direct absorption into the tissues; respiration). This type of measurement must be previously validated by a comparative analysis of biologically and chemically estimated bioavailable fractions. Some physical and chemical separations are not easy to perform, especially when it comes to differentiating between contaminants bound to colloids and truly dissolved contaminants in the water.

A biological measurement of bioavailability consists of measuring the various responses (usually toxic, but they can also be enzymatic, physiological, or related to bioaccumulation) induced by the pollutant on an organism or a population. The biological response is directly related to the bioavailable fraction of contaminants in the medium and not the total concentration. This indirect evaluation is the most relevant from a biological perspective, because it incorporates all the biological factors that may influence the bioavailability. In particular, the different routes of accumulation of pollutants in an organism are taken into account and undifferentiated. In the case of a biotest, the differences in resistance to contaminant between individuals are also integrated. The observation of a biological response is often accompanied by greater variability, in particular related to biological factors (e.g., physiology, internal response).

\section{Case study examples}

\section{Assessing copper bioavailability with influx in aquatic mosses}

In their study on the influence of organic matter on copper bioavailability, Ferreira et al. (2008) used the influx of metal into aquatic mosses as a biological endpoint to evaluate bioavailability. As illustrated in Figure 3, the relationship between bioavailable copper and the influx rate was first calibrated in mineral water without organic matter, in which total copper was directly proportional to bioavailable copper (the exposure medium remained constant). It was also observed that the addition of organic matter in the medium strongly reduced metal influx into the mosses. Measured influx in the same water containing organic matter could be used to estimate the bioavailable copper in this medium, using the "calibration curve" obtained in water only. 
Assessing benzo[a]pyrene bioavailability with bioaccumulation in daphnids

Similarly, Haitzer et al. (1999) and Gourlay et al. (2003) used bioaccumulation in aquatic organisms as an endpoint for benzo[a]pyrene bioavailability. Based on the assumption that only the truly dissolved compounds are bioavailable, the influence of organic matter on contaminant bioaccumulation was quantified using a "biologically-determined" partition coefficient $K D O C($ biol $)$ estimated from the reduction of bioaccumulation in the presence of organic matter:

$$
\frac{F_{D O M}}{F_{0}}=\frac{1}{1+K_{D O C}(\text { biol })[D O C]}
$$

$F_{D O M}$ and $F_{0}$ being respectively the concentration of benzo[a]pyrene in organisms exposed or not exposed to dissolved organic matter (Figure 4).

\section{Bioavailability in situ: interest of some passive sampling techniques}

In the 1990's, several sampling techniques were developed in order to better assess the bioavailable fraction of contaminants in the environment and improve risk analysis. Among them, passive sampling techniques gained importance, because of their ability to perform sampling in situ (see chapter on POCIS passive samplers in this book). Among the various samplers proposed, the diffusive gradient in thin films (DGT) developed by Davison and Zhang (1994) and the semi-permeable membrane device (SPMD) proposed by Huckins et al. (1993) have received much attention during the two last decades for their ability to sample bioavailable contaminants.

A DGT consists of a plastic support on which are mounted a phase for which the cationic metals have a very high affinity (Chelex resin), a hydrogel and a protective filter. During the immersion of the DGT, the accumulation of metals on the resin is proportional to the time and labile metal concentration in the medium. The metallic ions and complexes diffuse through the hydrogel and strongly bind to the resin. The metallic ion gradient that appears in the gel leads to the removal of weakly complexed metals. Hence, the labile metal concentration is close to inorganic metal concentration, since it comprises inorganic forms and metals weakly complexed to organic ligands. It is less than the total dissolved metal concentration measured in the water after filtration. Several studies have indicated that the DGTs allowed a first approximation of bioavailable metal concentration. Indeed, for copper and cadmium, the concentration of labile metal generally overestimates the concentration of bioavailable toxic levels (Tusseau-Vuillemin et al. 2004; Buzier et al. 2006), but is a much better estimation than the total dissolved metal concentration. Conversely, for more realistic levels of contamination, the bioavailable concentration of copper is slightly underestimated by a measure of labile copper (Figure 5).

A SPMD is made of a polyethylene tube containing a thin layer of triolein (Huckins et al. 1993). The membrane's hydrophobicity allows the solubilization of hydrophobic organic compounds in the membrane and their migration to the lipid phase. Once immersed in the aquatic environment from days to months, a SPMD accumulates hydrophobic organic substances (i.e., with octanol/water partitioning 
coefficients greater than 1000). The very small diameter of the pores in the membrane (10 $\mathrm{A})$ suggests that the sampled fraction is close to the freely dissolved fraction of contaminants. As is the case for biological membranes, it is assumed that compounds bound to dissolved organic matter in water are too large to cross the polyethylene membrane.

By comparing the fraction of PAHs available for accumulation in the presence of different types of organic matter, it was shown that the fractions sampled by the SPMD were similar to those accumulated in pelagic aquatic organisms (Gourlay et al. 2005). Consequently, the concentration estimated from SPMD accumulation can be considered as a better proxy of bioavailable hydrophobic compounds than their total dissolved concentration (Figure 6).

\section{Conclusions/prospects}

Taking into account bioavailability in environmental contamination issues has greatly helped our understanding of relationships between environmental contamination and observed toxic effects. Bioavailability models (BLM for metals, "free fraction" concept for hydrophobic substances) have been developed in the 1990's and several risk assessment methodologies suggest that they should be applied to better assess exposure concentrations.

It should be kept in mind that bioavailability models have usually been established under controlled and simplified conditions, with high, unrealistic concentrations and sometimes acute toxicity endpoints. There is growing evidence that bioavailability can differ in real environments, when considering low contamination concentrations and chronic toxicities. Most of the bioavailability models are currently being reviewed in light of recent experiments performed under more realistic conditions. Moreover, the application of models built from experiments on a limited number of species to field situations also needs to be further investigated.

If one assumes that bioavailability can be described by contamination partitioning in water only, without considering the dietary pathway, one could also underestimate actual risk. Depending on the feeding behaviour of target organisms, the trophic pathway may be more important than the waterborne route, particularly for organic contamination. Again, bioavailability of contaminants bound to particles remains poorly assessed and understood and merits further study. 


\section{References}

Anderson MA, Morel FMM, Guillard RRL (1978) Growth limitation of the coastal diatom by low zinc ion activity. Nature 276: 70-71.

Batley GE, Apte S, Stauber JL (2004) Speciation and Bioavailability of trace metals in water: progress since 1982. Australian Journal of Chemistry 57:903-919

Buzier R, Tusseau-Vuillemin MH, Mouchel JM (2006) Evaluation of DGT as a metal speciation tool in wastewater. Science of the Total Environment 358:277-285

Campbell PGC (1995) Interactions between trace metals and aquatic organisms : a critique of the Free Ion Activity Model. In: Tessier A, Turner DR (eds) Metal speciation and bioavailability in aquatic systems. John Wiley \& sons Ltd, pp 45-102.

Davison W, Zhang H (1994) In situ speciation measurements of trace components in natural waters using thin film gels. Nature 367:546-548

Degryse F, Smolders E, Merckx R (2005) Labile Cd Complexes Increase Cd Availability to Plants. Environmental Science \& Technology 40:830-836

Di Toro DM, McGrath JA, Hansen DJ, Berry WJ, Paquin PR, Mathew R, Wu KB, Santore RC (2005) Predicting sediment metal toxicity using a sediment biotic ligand model: Methodology and initial application Environmental Toxicology and Chemistry 24:2410-2427

Di Toro DM, Zarba CS, Hansen DJ, Berry WJ, Swartz RC, Cowan CE, Pavlou SP, Allen HE, Thomas NA, Paquin PR (1991) Technical basis for establishing sediment quality criteria for nonionic organic chemicals using equilibrium partitioning. Environmental Toxicology and Chemistry 10:1541-1583

Ehlers G, Loibner A (2006) Linking organic pollutant (bio)availability with geosorbent properties and biomimetic methodology: A review of geosorbent characterisation and (bio)availability prediction. Environmental Pollution 141:494-512

Ferreira D, Tousset N, Ridame C, Tusseau-Vuillemin M-H (2008) More than inorganic copper is bioavailable to aquatic mosses at environmentally relevant concentrations. Environmental Toxicology and Chemistry 27:2108-2116

Gourlay C, Miege C, Noir A, Ravelet C, Garric J, Mouchel J-M (2005) How accurately do semipermeable membrane devices measure the bioavailability of polycyclic aromatic hydrocarbons to Daphnia magna? Chemosphere 61:1734-1739

Gourlay C, Tusseau-Vuillemin M-H, Garric J, Mouchel J-M (2003) Effect of dissolved organic matter of various origins and biodegradabilities on the bioaccumulation of polycyclic aromatic hydrocarbons in Daphnia magna. Environmental Toxicology and Chemistry 22:1288-1294

Haitzer M, Hoss S, Traunspurger W, Steinberg C (1999) Relationship between concentration of dissolved organic matter (DOM) and the effect of DOM on the bioconcentration of benzo[a]pyrene. Aquatic Toxicology 45:147-158

Haitzer M, Höss S, Traunspurger W, Steinberg C (1998) Effects of dissolved organic matter on the bioconcentration of organic chemicals in aquatic organisms -a review. Chemosphere 378:13351362

Huckins JN, Manuweera GK, Petty JD, Mackay D, Lebo JA (1993) Lipid-containing semipermeable membrane devices for monitoring organic contaminants in water. Environmental Science and Technology 27:2489-2496

Landrum PF, Reinhold MD, Nihart SR, Eadie BJ (1985) Predicting the bioavailability of organic xenobiotics to Pontoporeia hoyi in the presence of humic and fulvic materials and natural dissolved organic matter. Environmental Toxicology and Chemistry 4:459-467

Meylan S, Behra R, Sigg L (2004) Influence of metal speciation in natural freshwater on bioaccumulation of copper and zinc in periphyton: a microcosm study. Environmental Science and Technology 


\section{8:3104-3111}

Morel FMM (1983) Principles of Aquatic Chemistry. John Wiley \& Sons, New York

Paquin PR et al. (2002) The Biotic Ligand Model : a historical overview. Comparative Biochemistry and Physiology Part C 33:3-35

Semple KT, Doick KJ, Jones KC, Burauel P, Craven A, H. H (2004) Defining bioavailability and bioaccessibility of contaminated soil and sediment is complicated. Environmental Science \& Technology 38:228A-231A

Slaveykova VI, Wilkinson KJ, Ceresa A, Pretsch E (2003) Role of fulvic acid on lead bioaccumulation by Chlorella kesslerii. Environmental Science \& Technology 37:1114-1121.

Tusseau-Vuillemin M-H, Gilbin R, Bakkaus E, Garric J (2004) Performance of diffusive Gradient in Thin Films in evaluating the toxic fraction of copper to Daphnia magna. Environmental Toxicology and Chemistry 23:2154-2161 


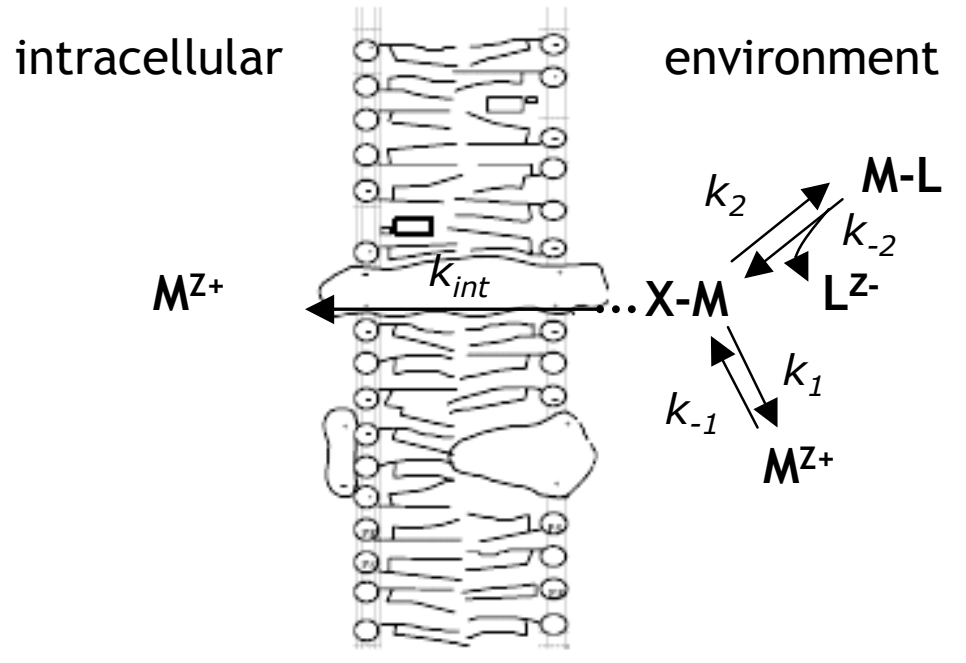

Figure 1. Schematic representation of the interaction of a metal $M$ with the cellular membrane. 


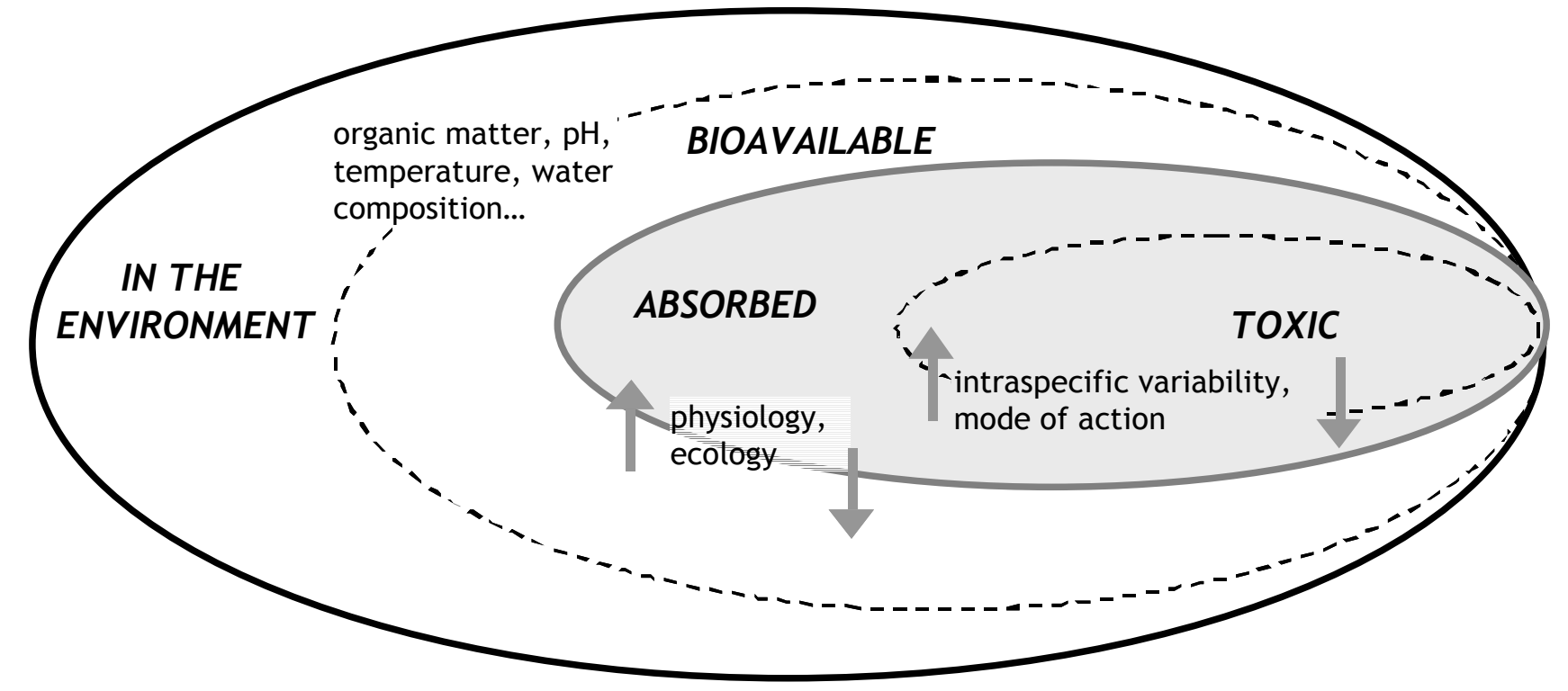

Figure 2. Schematic representation of the species of a contaminant regarding its potential toxicity. 


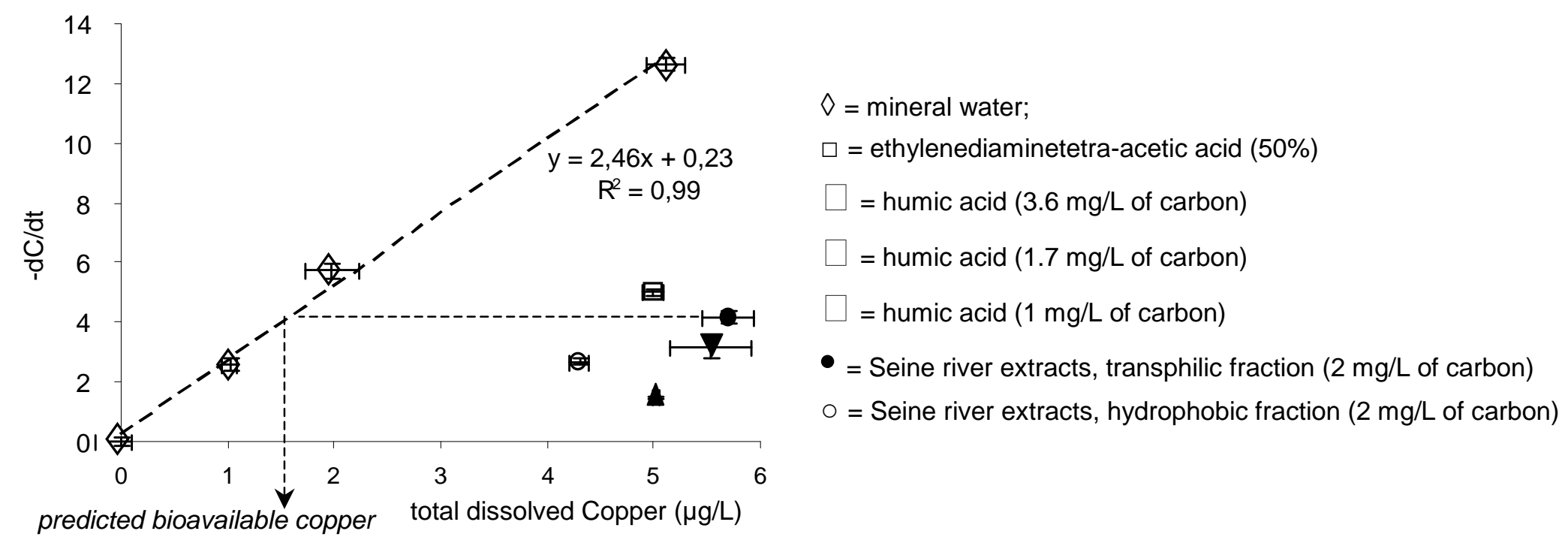

Figure 3. Rate of removal of dissolved copper from the exposure solution containing bryophytes, in mineral water (diamonds and straight line) or mineral water spiked with different types of organic matter. 


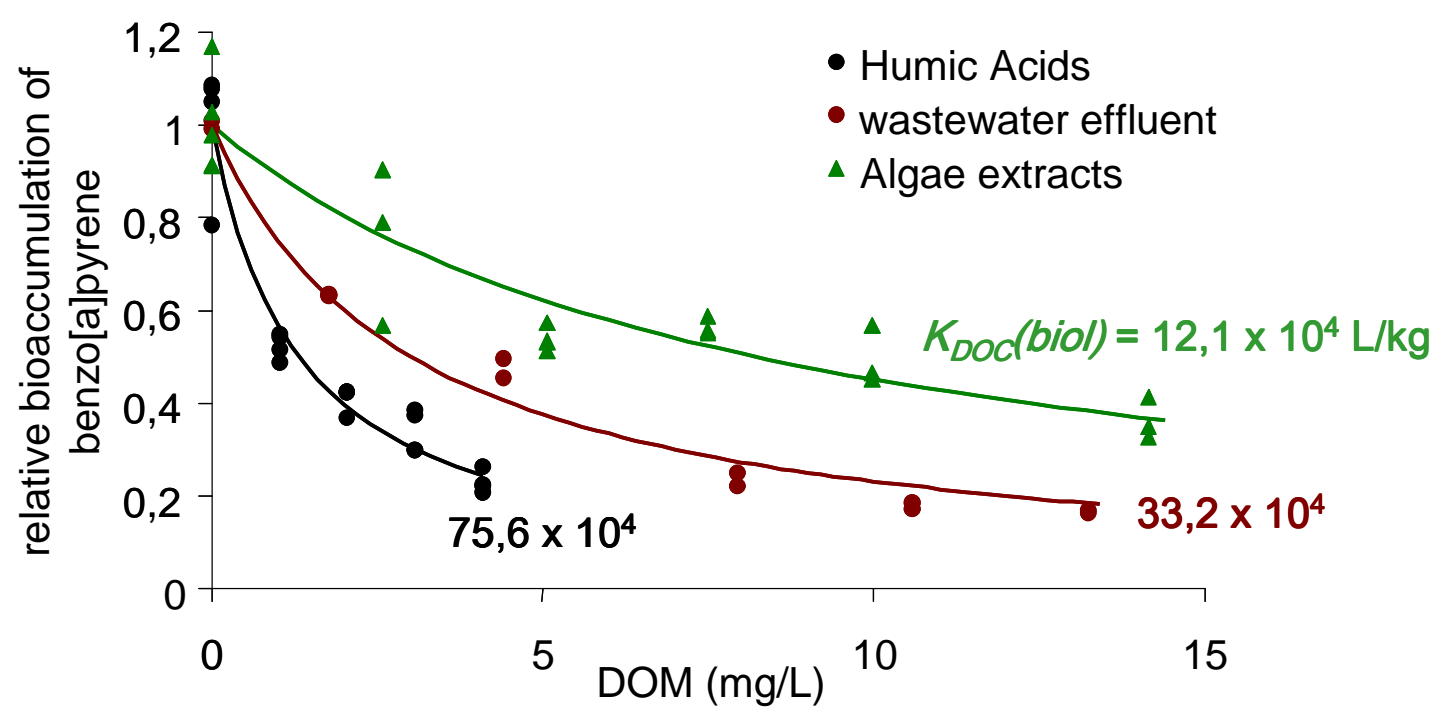

Figure 4. Relative bioaccumulation of benzo(a)pyrene with regards with spiking of the medium with dissolved organic matter of various origins. 


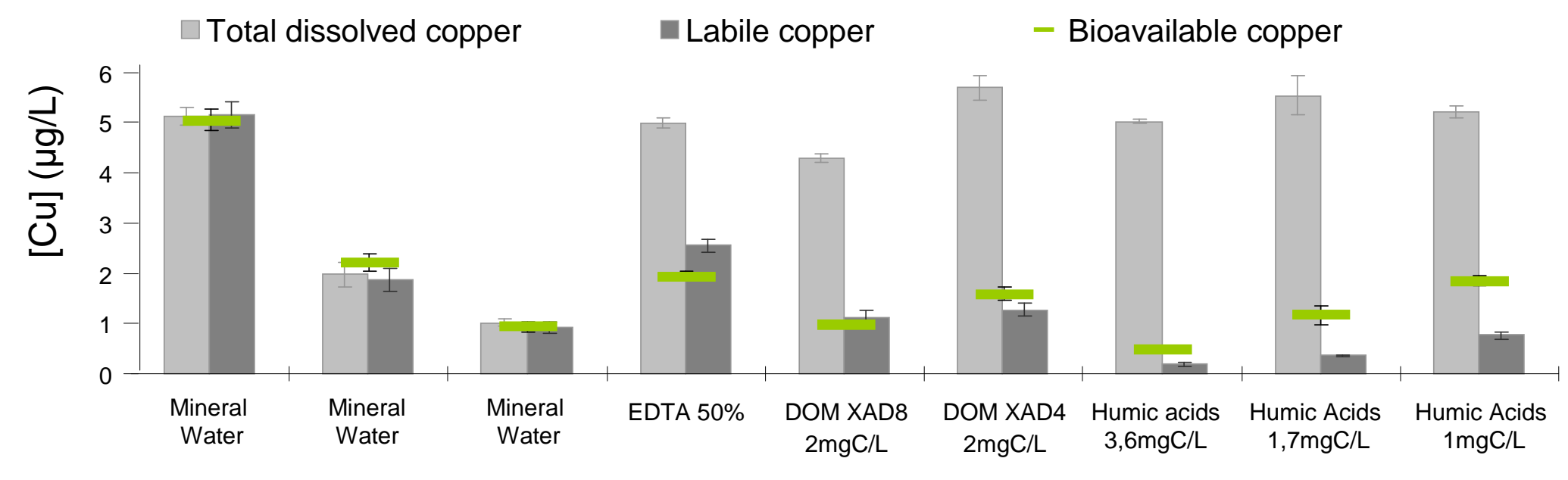

Figure 5. Comparison of total dissolved copper, labile copper (estimated with DGT), and bioavailable copper (with regards to bryophytes removal) in exposure medias spiked with various types of dissolved organic matter. 


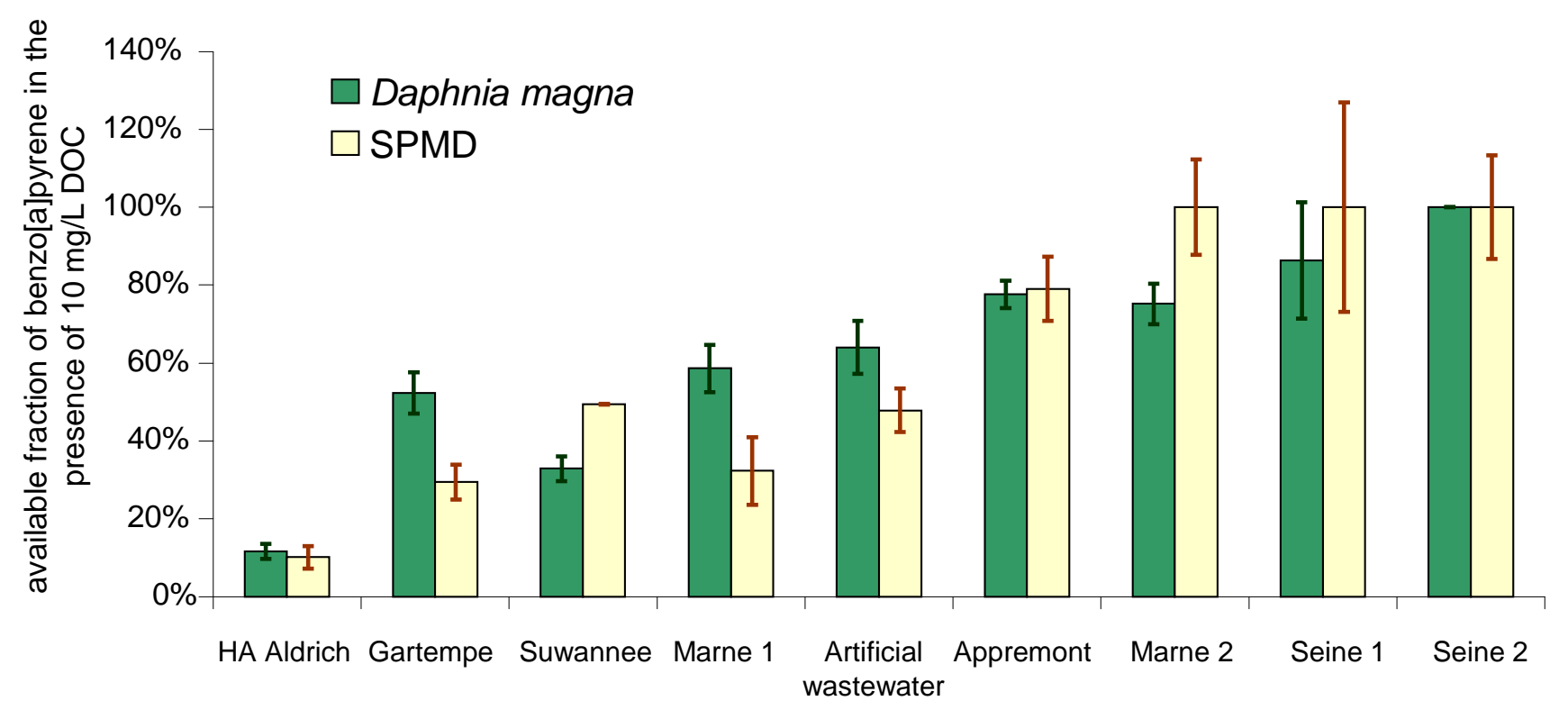

